\title{
Coronary angiography cannot be used to assess myocardial perfusion in patients undergoing reperfusion for acute myocardial infarction
}

"All knowledge, like all ignorance, deviates from the truth in an opportunistic direction"-Gunnar Myrdal from $A$ challenge to world poverty

Perfusion can be defined as tissue blood flow at the capillary level. There are two components of flow-volume and velocity. Blood flow is a volume of blood moving at a certain velocity. Approximately $8 \%$ of the left ventricular mass at rest is blood (the blood volume fraction of the myocardium ${ }^{1}$ ), about $90 \%$ of which is resident in capillaries. $^{2}$ The red blood cell velocity in the myocardium is approximately $1 \mathrm{~mm} / \mathrm{s}$ at rest. ${ }^{3}$ Myocardial blood flow at rest is approximately $1 \mathrm{ml} / \mathrm{min} / \mathrm{g}$.

Because there are no arteriovenous connections in the human heart, any blood entering the coronary artery will reach the capillaries under normal circumstances. Consequently, flow measured at any level-coronary arteries, arterioles, or capillaries-will reflect tissue perfusion. If flow is reduced in a coronary artery in the absence of infarction (such as in the setting of a critical stenosis) myocardial perfusion may still be normal at rest because of collateral flow. In this instance flow measured in the stenotic coronary artery will not correlate with either arteriolar or capillary flow (perfusion). Because the coronary artery from which the collaterals are emanating is now supplying flow to its own perfusion bed in addition to the bed with stenosis, its flow will increase. The flow measured in the small arterioles $(9-11 \mu \mathrm{m}$ diameter where radiolabelled microspheres lodge) and capillaries will underestimate total flow in this coronary artery since some of it is shunted to the other bed before reaching these levels.

The physiology of coronary blood flow is even more complex immediately after reperfusion in patients with acute myocardial infarction. In this situation, myocardial blood volume decreases in proportion to the extent of necrosis, ${ }^{4}$ while blood velocity generally increases above baseline pre-occlusion levels. ${ }^{4}$ The decrease in blood volume is caused by capillary injury and plugging resulting in "no-reflow" in the capillaries, " while the increase in blood velocity is secondary to reactive hyperaemia ${ }^{4}$ that lasts for several hours after reperfusion. The magnitude of hyperaemia depends on the degree of residual stenosis as well as the myocardial blood volume. If the residual stenosis is $<40-50 \%$ (which is readily accomplished in most patients with primary angioplasty and in a sizeable fraction of patients undergoing thrombolysis), then the degree of hyperaemia will depend primarily on the myocardial blood volume fraction.

At rest, the mean aortic pressure is approximately $90 \mathrm{~mm} \mathrm{Hg}$ while the capillary hydrostatic pressure is only $30 \mathrm{~mm} \mathrm{Hg}$. The reduction in perfusion pressure from the aorta to the capillaries is mediated in most part by coronary arterioles. ${ }^{6}$ During reactive or exogenously induced hyperaemia, coronary vessels become dilated in proportion to the amount of smooth muscle they possess. Thus, coronary arterioles are dilated the most and coronary venules also undergo some dilatation, resulting in reduction in total coronary resistance and increase in coronary blood flow. ${ }^{6}$ Because they do not possess smooth muscle, capillaries are not dilated, so myocardial blood volume fraction does not change in the presence of coronary vasodilators. ${ }^{13}$ Since their dimensions remain unaltered, capillaries offer the most resistance to flow during hyperaemia and also determine the maximal increase in hyperaemic coronary blood flow. ${ }^{7}$

Because capillaries are laid in parallel, the total capillary resistance is inversely related to the number of capillaries. Therefore, if no capillary damage (no necrosis) is incurred during coronary occlusion, the reactive hyperaemia after reflow would be expected to be maximal, provided that residual stenosis is $<40-50 \%{ }^{4}$ In the presence of myocardial necrosis the hyperaemic response will be attenuated in proportion to the degree of necrosis and capillary loss. ${ }^{4}$ Nonetheless, unless $>50 \%$ of the perfusion bed has undergone necrosis, flow will be above pre-occlusion resting levels. ${ }^{4}$ If $>50 \%$ of the perfusion bed has undergone necrosis then blood flow after reperfusion will be below normal levels and will bear an inverse relation to the degree of necrosis.

\section{Angiography immediately after reperfusion}

Based on these above considerations, what should be expected on coronary angiography in patients immediately after attempted reperfusion? The answer depends on the degree of dissociation between coronary blood flow and myocardial perfusion. If residual stenosis is minimal and the extent of necrosis is $<50 \%$ of the perfusion bed, reactive hyperaemia will be present and rapid disappearance of dye from the coronary arteries will be seen. Therefore, even with sizeable necrosis, coronary flow will be high and hence the disappearance rate of the dye will be rapid. It is for this reason that despite "no reflow" seen on myocardial contrast echocardiography (MCE), TIMI grade 3 flow can be present post-reperfusion. ${ }^{8}$ When $>50 \%$ residual stenosis is present, the hyperaemic response will be attenuated in proportion to the severity of stenosis. ${ }^{7}$ If necrosis is extensive ( $>50 \%$ of the perfusion bed), there should be no reactive hyperaemia and coronary blood flow will be reduced irrespective of the degree of residual coronary stenosis. In these cases, the rate of dye disappearance will be slow, which explains the occurrence of "no-reflow" on MCE in most patients with TIMI grades 1 and 2 flow. ${ }^{8}$

If radiographic dyes could measure capillary perfusion, then the presence of necrosis (and hence "no reflow") should be characterised by a hypolucent area on myocardial blush. Paradoxically, what we find is that a large area of necrosis is associated with hyperlucency, and that dye retention lasts longer with larger infarcts. How can this paradox be explained? MCE contrast agents consist of small gas-filled microbubbles which remain entirely within the intravascular space and possess a microvascular rheology 


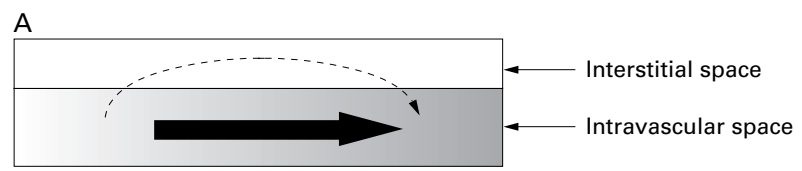

B

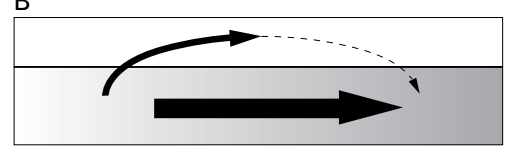

C

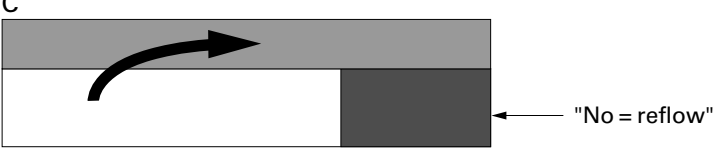

D

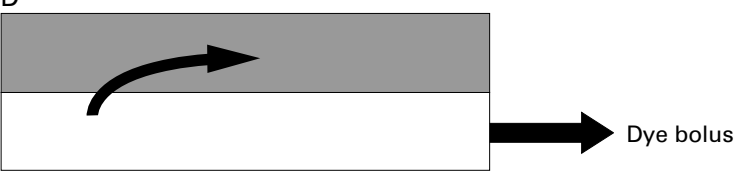

Figure 1 Schematic diagram of myocardial kinetics of radiographic and MRI contrast agents. (A) They transiently enter the extravascular space during normal circumstances. (B) The extravasation is increased as a result of increased endothelial permeability during microvascular injury. (C) The increased resistance in the distal capillary bed from the "no-reflow" phenomenon can accentuate this effect. (D) The duration of extravasation is prolonged beyond the intravascular duration of the dye when the size of the extravascular space increases as in myocardial oedema.

similar to that of red blood cells. ${ }^{9}$ These properties allow accurate assessments of both myocardial blood volume and blood velocity on MCE. ${ }^{9}$ In comparison, radiographic (and for that matter magnetic resonance imaging (MRI)) contrast agents are not pure intravascular tracers. They transiently exit the intravascular space before returning to the vascular space. ${ }^{10}{ }^{11}$ This extravascular sojourn partly contributes to the myocardial blush.

\section{Extravascular sojourn}

The magnitude of the extravascular sojourn depends on the degree of microvascular injury and the capillary resistance distal to the site of extravasation, whereas the duration of the sojourn is proportional to the size of the extravascular space. Under normal circumstances this sojourn is very brief (fig 1A). In the presence of microvascular injury, dye extravasation is accentuated because of increased capillary permeability (fig 1B). This extravasation cannot occur in the "no reflow" area since the dye never gets there. It occurs in the regions of the risk area which have been spared by necrosis, which is why the spatial extent of myocardial opacification on MRI immediately after reperfusion generally overestimates infarct size. ${ }^{12}$ The resistance of the extravascular space is lower than capillary resistance in the "no-reflow" zone distal to the site of extravasation, further accentuating the extravascular leak (fig 1C). If the extravascular space is large as occurs in myocardial oedema, the dye stays longer in this space (fig 1D) resulting in its prolonged retention. This principle is exploited to detect infarction with contrast MRI. Depending on the amount of myocardial oedema as well as the washout rate of the contrast agent from the extravascular space, the timing of imaging after contrast injection will determine the relation between the hyperlucent area and infarct size on MRI. ${ }^{13}$ The amount of myocardial oedema will depend on the type and size of infarction and other pre-existing myocardial disease, and therefore late phase imaging at one period of time will not always reflect actual infarct size.

But neither with myocardial blush on angiography nor with late phase imaging on MRI do we see myocardial perfusion, which is very low in regions of infarction. We see the sequelae of ischaemia (namely microvascular injury) as well as a byproduct of infarction (myocardial oedema and capillary plugging distal to the size of contrast extravasation). That these variables have some relation to infarct size and prognosis in patients with acute myocardial infarction should not be surprising. ${ }^{14}{ }^{15}$ Needless to say, an association is bound to exist between these variables and the extent of necrosis, but we are not measuring myocardial perfusion by assessing either of these two phenomena. It is time to stop saying so!

Cardiac Imaging Center,

SANJIV KAUL

Cardiovascular Division,

University of Virginia School of Medicine,

Charlottesville, Virginia, USA

sk@virginia.edu

I am grateful to Kevin Wei for providing a critical review of the manuscript and for executing the artwork. Supported in part by grants from the National Institutes of Health (3R01-HL48890 and 1RO1-HL65704), Bethesda, Maryland, USA

1 Wang $\mathrm{T}$, Wu X, Chung N, et al. Myocardial blood flow estimated by synchronous, multi-slice, high-speed computed tomography. IEEE Trans Med Imag 1989;8:70-7.

2 Kassab GS, Lin DH, Fung Y-CB. Morphometry of pig coronary venous system. Am f Physiol 1994;267:H2100-13.

3 Wei K, Jayaweera AR, Firoozan S, et al. Quantification of myocardial blood flow with ultrasound-induced destruction of microbubbles administered as a continuous infusion. Circulation 1998;97:473-83.

4 Villanueva FS, Glasheen WP, Sklenar J, et al. Characterization of spatial patterns of flow within the reperfused myocardium using myocardial contrast echocardiography: implications in determining the extent of myocardial
salvage. Circulation 1993;88:2596-606.

5 Kloner RA, Ganote CE, Jennings RB. The "no-reflow" phenomenon after temporary coronary occlusion in the dog. F Clin Invest 1974;54:1496-508.

6 Chilian WM, Eastham CL, Marcus ML. Microvascular distribution of coronary vascular resistance in beating left ventricle. Am $\mathcal{f}$ Physiol onary vascular resi

7 Jayaweera AR, Wei K, Coggins $M$, et al. Role of capillaries in determining CBF reserve: new insights using myocardial contrast echocardiography. $A m$ f Physiol 1999;277:H2363-72.

8 Ito $\mathrm{H}$, Okamura A, Iwakura K, et al. Myocardial perfusion patterns related to thrombolysis in myocardial infarction perfusion grades after coronary angioplasty in patients with acute anterior wall myocardial infarction. Circulation 1996;93:1993-9.

9 Keller MW, Segal SS, Kaul S, et al. The behavior of sonicated albumin microbubbles within the microcirculation: a basis for their use during myocardial contrast echocardiography. Circ Res 1989;65:458-67.

10 Canty JM, Judd RM, Brody AS, et al. First-pass entry of nonionic contrast agent into the myocardial extravascular space. Effects on radiographic estimates of transit time and blood volume. Circulation 1991;84:2071-81.

11 Laarson HBW, Stubgaard M, Sondergaard L, et al. In vivo quantification of the unidirectional influx constant for Gd-DTPA diffusion across the myocardial capillaries with MR imaging. F Magn Reson Imaging 1994;4:433-40.

12 Rogers WJ, Kramer CM, Geskin G, et al. Early contrast-enhanced MRI predicts late functional recovery after reperfused myocardial infarction. Circulation 1999;99:744-50.

13 Kramer CM, Rogers WJ Jr, Mankad S, et al. Contractile reserve and contrast uptake pattern by magnetic resonance imaging and functional recovery after reperfused myocardial infarction. F Am Coll Cardiol 2000;36:1835-40.

14 Gibson CM, Cannon CP, Murphy KA, et al. Relationship of TIMI myocardial perfusion grade to mortality after administration of thrombolytic drugs. Circulation 2000;101:125-30.

15 Appleby MA, Angeja BG, Dauterman K, et al. Angiographic assessment of myocardial perfusion: TIMI myocardial perfusion (TMP) grading system. Heart 2001;86:485-6. 